\title{
Close contact infection dynamics over time: insights from a second large-scale social contact survey in Flanders, Belgium, in 2010-2011
}

Thang Van Hoang ${ }^{1 *}$ (D), Pietro Coletti ${ }^{1}$, Yimer Wasihun Kifle $^{2}$, Kim Van Kerckhove ${ }^{1}$, Sarah Vercruysse ${ }^{1}$, Lander Willem ${ }^{3}$, Philippe Beutels ${ }^{3,4}$ and Niel Hens $s^{1,3}$

\begin{abstract}
Background: In 2010-2011, we conducted a social contact survey in Flanders, Belgium, aimed at improving and extending the design of the first social contact survey conducted in Belgium in 2006. This second social contact survey aimed to enable, for the first time, the estimation of social mixing patterns for an age range of 0 to 99 years and the investigation of whether contact rates remain stable over this 5-year time period.

Methods: Different data mining techniques are used to explore the data, and the age-specific number of social contacts and the age-specific contact rates are modelled using a generalized additive models for location, scale and shape (GAMLSS) model. We compare different matrices using assortativeness measures. The relative change in the basic reproduction number $\left(R_{0}\right)$ and the ratio of relative incidences with $95 \%$ bootstrap confidence intervals $(\mathrm{BCl})$ are employed to investigate and quantify the impact on epidemic spread due to differences in sex, day of the week, holiday vs. regular periods and changes in mixing patterns over the 5-year time gap between the 2006 and 2010-2011 surveys. Finally, we compare the fit of the contact matrices in 2006 and 2010-2011 to Varicella serological data.

Results: All estimated contact patterns featured strong homophily in age and sex, especially for small children and adolescents. A 30\% (95\% BCl [17\%; 37\%]) and 29\% (95\% BCI [14\%; 40\%]) reduction in Ro was observed for weekend versus weekdays and for holiday versus regular periods, respectively. Significantly more interactions between people aged 60+ years and their grandchildren were observed on holiday and weekend days than on regular weekdays. Comparing contact patterns using different methods did not show any substantial differences over the 5-year time period under study.

Conclusions: The second social contact survey in Flanders, Belgium, endorses the findings of its 2006 predecessor and adds important information on the social mixing patterns of people older than 60 years of age. Based on this analysis, the mixing patterns of people older than 60 years exhibit considerable heterogeneity, and overall, the comparison of the two surveys shows that social contact rates can be assumed stable in Flanders over a time span of 5 years.
\end{abstract}

Keywords: Contact rates, Contact behaviour, Mixing patterns, Behavioural changes, Infectious diseases

\footnotetext{
*Correspondence: vanthang.hoang@uhasselt.be

'I-Biostat, Data Science Institute, Hasselt University, Martelarenlaan 42, 3500

Hasselt, Belgium

Full list of author information is available at the end of the article
}

(c) The Author(s). 2021 Open Access This article is licensed under a Creative Commons Attribution 4.0 International License, which permits use, sharing, adaptation, distribution and reproduction in any medium or format, as long as you give appropriate credit to the original author(s) and the source, provide a link to the Creative Commons licence, and indicate if changes were made. The images or other third party material in this article are included in the article's Creative Commons licence, unless indicated otherwise in a credit line to the material. If material is not included in the article's Creative Commons licence and your intended use is not permitted by statutory regulation or exceeds the permitted use, you will need to obtain permission directly from the copyright holder. To view a copy of this licence, visit http://creativecommons.org/licenses/by/4.0/. The Creative Commons Public Domain Dedication waiver (http://creativecommons.org/publicdomain/zero/1.0/) applies to the data made available in this article, unless otherwise stated in a credit line to the data. 


\section{Background}

Infectious diseases and, more specifically, airborne infections can be transmitted between hosts via close contact interactions; therefore, quantifying such interactions provides important information for properly modelling infectious disease transmission. In recent years, we have witnessed a paradigm shift with respect to this: whereas at the start of this century, mathematical models relied on simplifying assumptions such as homogeneous mixing or on using mathematically convenient "Who Acquires Infection From Whom" constructs [1], a vast number of studies now rely on the use of social contact data [2-8].

The literature on social contact surveys has shown how human interactions are heterogeneous in nature and present a large degree of homophily in terms of age $[9,10]$ and sex [11]. The information coming from social contact surveys is therefore usually summarized in what is called the social contact matrix, quantifying the average number of contacts made between individuals within and between given age classes. Using the social contact hypothesis [2], i.e. assuming that transmission rates are proportional to social contact rates, these data-driven mixing patterns have been implemented into models of infectious disease transmission showing good correspondence to (sero)prevalence data; see, e.g., [4, 9, 12].

Social contact survey data allow for an exploration of contact rate patterns stratified by age, sex, and location, which helps to better describe the structure of the transmission network $[13,14]$. However, a systematic review by Hoang et al. (2019) [10] showed that half of the social contact surveys before 2019 used convenience sampling, while quite a few surveys were conducted in specific settings, e.g., schools or universities, and/or focus on specific target groups; thus, it is impossible to extrapolate the results to an entire population. Even in population-based social contact surveys with representative samples, two problems might still exist: the sample does not cover all age ranges of the population, or the number of elderly participants is insufficient for investigating mixing patterns of these people. Indeed, no study reported the contact rates of people up to 99 years old.

Particular attention has been devoted to behavioural changes with respect to individual health status (e.g., being ill [6, 15-17]), weather conditions [5] or day of the week (weekday or weekend in holiday/non-holiday or regular periods $[9,18-22])$ - hereafter referred to as microscopic time settings, and how these affect disease dynamics [6, 8, 19, 23, 24].

The use of social contact data to inform modelling has become so prominent in recent works that it has also been applied to settings for which social contact studies are not available, leading to the question of how social contact matrices should be projected onto other geographical areas and in time [7, 25-27]. However, to the best of our knowledge, there has been no empirical assessment of whether mixing patterns change over longer time periods (e.g., years) within a particular population and how this should be taken into account when projecting social contact matrices. We will refer to these as macroscopic time changes to mark the difference with microscopic time changes.

A first population-based social contact survey in Belgium was conducted in 2006, and its results were reported in $[9,19,28]$, in which the impact of microscopic time changes on the contact mixing pattern was investigated, although this study was not designed for doing so. A second population-based survey in Belgium was conducted 5 years later in 2010-2011. This survey was conceived as an improvement over the 2006 survey, with a larger sample size covering a wider age range of participants and a better distribution of surveyed participants over four different time settings (weekday/weekend days in regular/holiday periods).

In this work, we aim to describe and analyse the social contact survey in Flanders, Belgium from 2010-2011 by accounting for the mixing patterns of people 0-99 years of age, with a particular focus on elderly people. We study both the impact of microscopic and macroscopic time changes on contact patterns, and we assess whether the contact rates remain stable over 5 years timespan.

\section{Data}

Social contact survey in 2006: This survey was part of the POLYMOD project, in which social contact surveys were conducted in 8 European countries in 2005-2006 [9]. In the social contact survey conducted in Belgium in 2006 , a total of 750 participants were recruited by random digit dialing on land lines. The survey sample covered all three regions in Belgium; i.e. the Flemish, Walloon and Brussels-Capital regions, with quota sampling by age, sex, and region, making it representative for the whole Belgian population. Each participant was asked to fill in a background questionnaire and a paper diary in which they record their contacts over 2 days: one randomly assigned weekday and one randomly assigned weekend day. Two types of contacts were defined: (1) two-way conversations during which at least three words were spoken and (2) contacts that involved skin-to-skin touching. Information recorded in the diary included sex and the exact age or presumed age interval of each contacted person over the entire day. Contact features included frequency, location and duration. If participants established more than 20 professional contacts per day, then they only had to provide an estimated number of professional contacts and the age interval(s) with whom they interacted most. Contact information (e.g., contact age or contact duration) was then imputed for such contacts. More details can be 
found in [28]. We will refer to those contacts as additional professional contacts.

Social contact survey in 2010-2011: This survey was conducted between September 2010 and February 2011 in Flanders (including the Flemish and Brussels-Capital regions) in Belgium using an adapted version of the diaries used in the first Belgian survey in 2006. Three different types of diaries were designed to adapt to the age of participants: one for children (less than 13 years old), which was completed by a proxy, e.g., parents or school teachers; one for people aged 13-60 years and one for people aged $60+$ years, which could also be filled out by a proxy. A total of 1,774 participants were recruited by random digit dialing on mobile phones and landlines, with quota sampling by age, sex and geographical location. The contact definitions were the same as those used in the 2006 survey. Participants were asked to complete a background survey and record their social contacts in a paper diary during one randomly assigned day. Information on additional professional contacts was imputed the same way as done for 2006 data. Compared with the 2006 survey, the 2010-2011 survey explored more features that might influence the number of contacts recorded: the health conditions of participants, time use, distance from home, animal ownership and touching. To date, the impact of animal ownership and touching on social contacts has been investigated [29], so has the impact of weather on social contacts [5]. Of particular focus were people aged 60 years and above; i.e., participants up to 99 years of age were recruited, and information about contact frequency with children and grandchildren and residence size for elderly people living in nursing/elderly homes was recorded.

The design of the 2010-2011 survey is similar to that of 2006, with the difference being that in 2010-2011, participants reported information for only one day, whereas in 2006, information was collected for two days. Since participants have been shown to be influenced by fatigue in reporting on multiple days $[10,22]$, only data on the first day of the 2006 survey was used for comparison with the 2010-2011 survey in this work. Additionally, we extracted the 511 participants recruited in Flanders from the 2006 survey to be in line with the study population in the 2010-2011 survey. In the 2010-2011 survey, 15 cases were removed since the diaries were unreliable (many answers left blank, incoherent answers, etc.). We also excluded 46 people living in an elderly/nursing home and explored the contact patterns of these people separately. In addition, 6 people aged 90 and older ( 4 in the age group $[90 ; 95)$ and 2 in the age group $[95 ; 100))$ for reasons of data sparsity, e.g. when investigating the impact of microscopic time differences on the age group-specific degree distribution or sex differences in mixing patterns. As a result, the final sample for the analysis of the 2010-2011 survey is 1,707 participants. We defined four microscopic time settings: regular weekdays, regular weekends, holiday weekends, and holiday weekdays. Holiday periods include both public holidays and weekends inside or adjacent to these holidays. More details on the number of participants by age and microscopic time in both surveys can be found in Additional file 1 Table S1. The datasets of both surveys are available online within the social contact data sharing initiative [30] and the SOCRATES platform [14].

\section{Methods}

We started with a descriptive analysis to explore the sociodemographic characteristics of survey participants and features of their reported contacts for the contact survey in 2010-2011. Subsequently, data mining techniques were used to explore associations among variables of interest and contact profiles of survey participants. We then investigated the factors associated with the number of contacts, differences in sex in mixing patterns and the impact of holidays and weekends as a proxy for the impact of school closure on disease transmission. We ended with the comparison between the contact surveys from 2006 and 2010-2011 using different measures.

\section{Data mining techniques}

We used two unsupervised learning methods: association rules and clustering. Association rules were used to assess the possible associations pertaining to contact features, e.g., type of contact (close or non-close), duration and frequency of contacts, ..., using support, confidence and lift values as measures of interestingness $[19,31]$ (see Additional file 2 for Additional information). Rules were considered of interest only when the support value exceeded $1 \%$, equivalent to at least 3142 contacts involved in constructing the rules. The threshold for the confidence was $70 \%$, and rules with greater lift indicates stronger association. In addition to association rules, we investigated contact profiles using a clustering method. The contact profiles were defined by (1) the number of contacts per survey participant in six different locations (home, work, school, leisure, transport and other), (2) characteristics of participants (age and sex) and (3) time indicators (weekday/weekend and regular/holiday period). Clustering was implemented using the daisy function in the $\mathrm{R}$ package "Cluster" [32] and using the Gower distance, allowing for mixed types of variables. We visualized the clusters by projecting them into a low-dimensional space using a dimension reduction technique known as the $t$ distributed stochastic neighbor embedding [33]. Contacts reported at different locations were attributed to just one location using the following hierarchy: (1) contacts at home, (2) contacts at work, (3) contacts at school, (4) 
contacts during leisure time, (5) contacts in other locations and (6) contacts in transportation.

\section{Modelling the number of contacts Degree distribution}

We considered both physical and non-physical contacts, including additional professional contacts reported by participants. We modeled the number of contacts using a weighted negative binomial regression model to account for over-dispersion. Socio-demographic characteristics, the health status of participants and microscopic time settings (weekdays/weekends and regular/holiday period) were included as possible determinants (Descriptive statistics see Additional file 1). In addition, diary weights computed from age and household size were used to account for under-/over-sampling over participant features $[9,28]$. We performed variable selection using a random forest analysis [31] and the likelihood ratio test (LRT). Interactions between age and microscopic time settings were retained, as they were the two most significant determinants of the number of contacts reported in the literature [10].

\section{Estimating age-specific contact rates}

We defined the age-specific number of contacts $y_{i j r}$ as the number of contacts made by the $r^{t h}$ participant in age class $i$ with people in age class $j$ per day $(i, j=1, \cdots, J ; r=$ $1, \cdots, n_{i}$ ), where $J$ is the number of age classes, and $n_{i}$ is the number of participants in age class $i$.

The age-specific number of contacts $y_{i j r}$ was assumed to follow a negative binomial distribution to account for over-dispersion [9]. This distribution is defined as $y_{i j r} \mid \boldsymbol{x} \sim$ $N B\left(m_{i j}, \kappa_{i j}\right)$ for a vector of covariates $\boldsymbol{x}$, in our case the age of the participant $x_{1 i}$ and the age of the contact $x_{2 j}$. The mean and variance of this distribution are defined as $m_{i j}$ and $m_{i j}+\kappa_{i j} *\left(m_{i j}^{2}\right)$, respectively, where $\kappa_{i j}$ is the overdispersion parameter. To model the age-specific number of contacts, we applied generalized additive models for location, scale and shape (GAMLSS). This allowed for modelling both the mean and variance (over-dispersion) parameters of the negative binomial distribution over participants' age $x_{1 i}$ and contacts' age $x_{2 j}$. We refer to Additional file 3 and [34] for details about the GAMLSS. When estimating the social contact matrix $C$, the reciprocal nature of making contact was taken into account, as $m_{i j} N_{i}=m_{j i} N_{j}$, where $N_{i}$ is the population size in age class $i$ (obtained from demographic data) [35]. Based on $m_{i j}$ and $N_{i}$, the reciprocal contact rates $c_{i j}$ can be obtained by $c_{i j}=\frac{\left(m_{i j} N_{i}+m_{j i} N_{j}\right)}{\left(2 N_{i} N_{j}\right)}$.

For all quantities of interest, introduced in this and following subsections, we used a non-parametric bootstrap of participants, to obtain $95 \%$ percentile bootstrap confidence intervals (BCIs) [36].
Measures of comparison between different mixing patterns We used four different measures of comparison: two for measuring assortativeness, the relative change in $R_{0}$ and the relative incidence (RI). We measured the assortativeness of contacts by age using 2 different indices. The first index was Gupta's Q [37], ranging from 0 (= homogeneous mixing) to 1 (= completely assortative mixing). The second index was $I_{s}^{2}$, as proposed in [38] ranging from 0 (= perfect assortativity) to 1 (= homogeneous mixing).

The third measure was based on the basic reproduction number $R_{0} . R_{0}$ was calculated as the dominant eigenvalue of the next generation matrix $G$. Assuming the age-specific transmission rates $\beta(i, j)$ were proportional to the age-specific social contact rates $c(i, j)$ (also known as the social contact hypothesis [2]), the ratio of dominant eigenvalues of the next generation matrix was the relative change in basic reproduction number using different mixing patterns. Lastly, the ratio of relative incidences (RRI) was used for comparison. The expected age-specific RI in the population during the exponential phase was given by the leading right eigenvector of the next-generation matrix [39].

For more details, we refer to Additional file 3.

\section{Investigating sex differences in mixing patterns}

To gain insights into possibly different mixing behaviour between males and females, we estimated the age-specific average number of contacts using the GAMLSS approach (as previously introduced) for all four combinations of sex interactions (male-male, female-female, male-female and female-male). We used assortativeness measures $\left(I_{s}^{2}\right.$ and $\mathrm{Q}$ indices), and the RRI to study differences between matrices.

\section{Investigating the impact of school closure on disease transmission}

We estimated the impact of school closure based on social contact data using contact rates from holidays and weekend days as a proxy and compared them with contact rates from regular weekdays. We used the changes in $R_{0}$ and the RRI to quantify these differences.

\section{Comparing the contact surveys in 2006 and 2010-2011}

The designs of the contact surveys in 2006 and 20102011 were similar (see Data section). However, the 2006 survey did not cover age groups up until 90 years of age or sufficiently cover all four microscopic time settings (see Additional file 1 Table S1). Therefore, for the comparison of contact matrices between 2006 and 20102011, we only used participants less than 65 years old and merged weekend-regular and weekend-holiday into one "weekend" category to overcome the data sparsity problem. 


\section{Degree distribution of contacts from aggregated data}

To investigate potential determinants for the number of contacts, we combined data from 2006 and 2010-2011 using a survey indicator variable as an additional determinant (Table 1). We first applied a random forest analysis [31] to identify main predictors, after which we modeled the aggregated number of contacts via a GAMLSS model assuming a negative binomial distribution for the response variable. Model selection was performed using the likelihood ratio test for mean and dispersion parameters.

\section{Comparing contact matrices}

We first visually compared contact matrices in 2006 and 2010-2011 for the different microscopic time settings. Both assortativeness indices, the change in $R_{0}$ and the $R R I$ were used to compare mixing patterns. Furthermore, we used the ratio of transmission rates that allows for the direct comparison of contact rates between contact matrices (a cell-wise comparison). Lastly, we applied the methods outlined in [4] to use social contact matrices to fit Varicella zoster virus (VZV) serological data from Belgium based on the social contact hypothesis (i.e., constant proportionality, [2]), with both contact data sets separately and compared the results.

\section{Results}

\section{Socio-demographic characteristics of participants}

A total of 1,707 participants ( $46 \%$ males and $54 \%$ females) were used for the analysis of the 2010-2011 dataset, of whom 1,011 reported their contacts on regular weekdays, 257 on regular weekend days, 286 on weekdays during public holidays and 151 on weekend days during or adjacent to public holidays ( 2 cases did not indicate the date). The average participant age was 38 years, and participants younger than 18 years of age accounted for $22 \%$ of the sample. The average household size was 3, ranging from 1 to 11; participants with household sizes of $2-4$ accounted for nearly $75 \%$ of the sample size, while only $13 \%$ of participants lived in households with more than 4 residents. Approximately $21 \%$ of the participants were still students, $48 \%$ had a job, $13 \%$ were retired and

Table 1 List of the common determinants selected from the two social contact surveys

\begin{tabular}{ll}
\hline Variable & Categories \\
\hline Age & {$[0,5),[5,10),[10,15),[15,20),[20,25),[25,30),[30,35)$,} \\
& {$[35,40),[40,45),[45,50),[50,55),[55,60),[60,65)$} \\
sex & female, male \\
Hh-size & $1,2,3,4, \geq 5$, missing \\
Microscopic time & holiday-weekday, regular-weekday, weekend \\
Macroscopic time & $2006,2010-2011$ \\
\hline
\end{tabular}

approximately $11 \%$ were at home or unemployed. Nearly two-thirds of working participants were office clerks, $12 \%$ were manual workers, and only $6 \%$ were self-employed (Table S2 in Additional file 1).

\section{Daily number of contacts and contact features}

A total of 31,423 contacts including 8,527 imputed professional contacts were recorded by the 1,707 participants: the highest number of contacts reported by one participant was 364 (mostly professional contacts), and the lowest was zero (15 cases). The median number of reported contacts were 12 (interquartile range (IQR):[6; 21]). Participants reported an average of 18.4 contacts per day $(\mathrm{SD}=24.3)$, skewed in distribution. This reduced to 13.4 $(\mathrm{SD}=10.8)$ when professional contacts were excluded. By adjusting for the age and household size of Flanders and type of day (weekdays/weekend days), the average number of contacts was 17.2 (12.0 when excluding professional contacts).

Nearly half the number of contacts involved touching (with missing information in 345 cases). More than 10\% of all contacts were with household members. Daily contacts accounted for nearly one-third of the total number of contacts, while only $10 \%$ were first-time contacts. Short contacts (less than 5 minutes) made up approximately 15\% of the total number of contacts; long contacts (longer than 1 hour) constituted nearly half of the total number of contacts. Nearly two-thirds of all reported contacts were made at home, work and school, while contacts at multiple locations accounted for only $6 \%$ of all contacts.

\section{Data mining techniques}

The association rules with the highest lift value are presented in Table S1 in Additional file 2. Seventy-four percent of daily contacts lasting longer than 4 hours involved skin-to-skin touching. In contrast, $81 \%$ of the contacts lasting less than 5 minutes with non-household members were usually non-physical contacts. Contacts with household members were the most influential factor in determining whether contacts occurred on a daily basis. Contacts lasting longer than 4 hours, occurring on weekdays in a regular period, tended to occur on a daily basis (71\%)

In the clustering analysis, the largest silhouette width was obtained for six clusters (Figure S1 in Additional file 2). The cluster sizes ranged from 151 participants (cluster 5) to 443 participants (cluster 1). All clusters presented a strong connection with the microscopic time settings, including participants from only weekdays/weekends or regular days/holidays (Table S2 in Additional file 2). Some clusters were easy to interpret when looking at the cluster members' features. Cluster 2, for example, was composed of participants whose average age was 9 years, with a large number of contacts at school, i.e., school-aged 
children. Cluster 4 included participants with an average age of 39 years and a large number of contacts at work, i.e., working-age adults. Other clusters presented less specific contact patterns but still exhibited a strong connection with microscopic time settings. Specifically, cluster 1 included participants who had a low number of contacts in all locations on regular weekdays. Participants in this cluster had the highest average age (51 years) and can be interpreted as being socially non-active. Cluster 3 included participants surveyed on the weekend and regular period, with few contacts at work and school but the highest number of contacts during leisure activities and at "other" locations. Cluster 5 contained participants surveyed in the weekend and holiday period, with no contacts at school, few contacts at work and most contacts at home and in "other" locations. Cluster 6 consisted of participants surveyed in the weekday and holiday period, with an average of 6 contacts at work, a very low number of contacts during leisure activities and transportation (see Additional file 2 Table S2 and Figure S2).

\section{Degree distribution for the social contact survey in 2010-2011}

Figure 1 shows the results of the weighted negative binomial model for the number of contacts. Several sociodemographic indicators had a significant effect on the number of contacts: age, household size, place of living and the use of public transportation. It is noted that occupation and education have been excluded from the model, as they strongly correlated with age, and that for participants younger than 13 years, the mother's educational level was used instead of that of the participant. Other variables (sex, animal ownership, health states regarding self-care and pain) were also excluded after model selection was performed (see Additional file 3 Table S1 and Figure S1). The interactions between age and microscopic time indicators were highly significant. In Fig. 1, we compared the number of contacts among age groups in each time setting: for weekdays in the regular period, participants older than 5 years of age had a higher number of contacts than children aged $0-5$ years, except the 15-25 age group, and people older than 60 years who had the lowest number of contacts. For holiday weekdays, people between 40 and 50 years of age had the highest number of contacts, while other age groups showed no difference in the number of contacts relative to the youngest age group (0-5 years). The number of contacts of children aged $0-5$ decreased by $25 \%, 46 \%$ and $54 \%$ with respect to regular weekdays during holiday weekdays, regular weekends and holiday weekends, respectively. During regular periods, people aged 75-90 had almost double the contacts during weekends with respect to weekdays. A higher number of contacts was observed in participants living in larger households and those using public transportation. The number of contacts was $41 \%$ lower in those who felt ill on the survey day. The reported health indicators showed that feeling anxiety and having problems in carrying out daily activities had a negative effect on the number of contacts, reducing them by $18 \%$ and $35 \%$, respectively.

\section{Contacts of children, working people and elderly people}

Some variables were only present in diaries for children (less than 13 years old), adults or people older than 60 years of age, so these variables were not included in the previous model (Fig. 1). Children attending preschools or schools had more contacts than children at childcare outside home and young children who were kept at home ( $\mathrm{P}<0.001$, Kruskal-Wallis test). It is also observed that there was no difference in the number of school contacts among different class sizes $(\mathrm{P}=0.40$, Kruskal-Wallis test). Children less than 3 years old had nearly $40 \%$ of their contacts at home. School contacts made up $44 \%$ of the number of contacts for participants younger than 18 years old, and this figure increased to $60 \%$ on weekdays and during regular periods. People with a job had a much higher number of contacts than those who were retired or currently unemployed/job seeking. Working people had most contacts at work (63\% on a random day and $71 \%$ on regular weekdays). When considering occupation, the number of contacts of office clerks was observed to be significant higher than those of people with other occupations.

Modelling the number of contacts for participants older than 60 years who were not living in an elderly/nursing home (Additional file 3 Table S2) shows that drinking status had no significant effect on the number of contacts and neither did having children or grandchildren. Smokers had fewer contacts than those who are non-smokers or used to smoke. Elderly people who experienced problems in performing their daily activities had fewer contacts than those who did not experience problems (relative number of contacts (RNC): 0.68; 95\% CI [0.51;0.91]). The effect of microscopic time settings was significant: more contacts were observed in weekend-regular periods than in weekday-regular periods. The majority of elderly people who had children and/or grandchildren reported having contacts with their children and grandchildren a few times per week or month. Figure 2 describes the social interaction of people aged $60+$ years with other age groups. People aged 61-79 years had the highest number of contacts with age group $[40,60)$, which may describe the mixing pattern of people from 2 generations. Interaction between people aged $60+$ years and young children/teenagers was significantly higher on holiday-weekdays and weekend days than on regular-weekdays. 


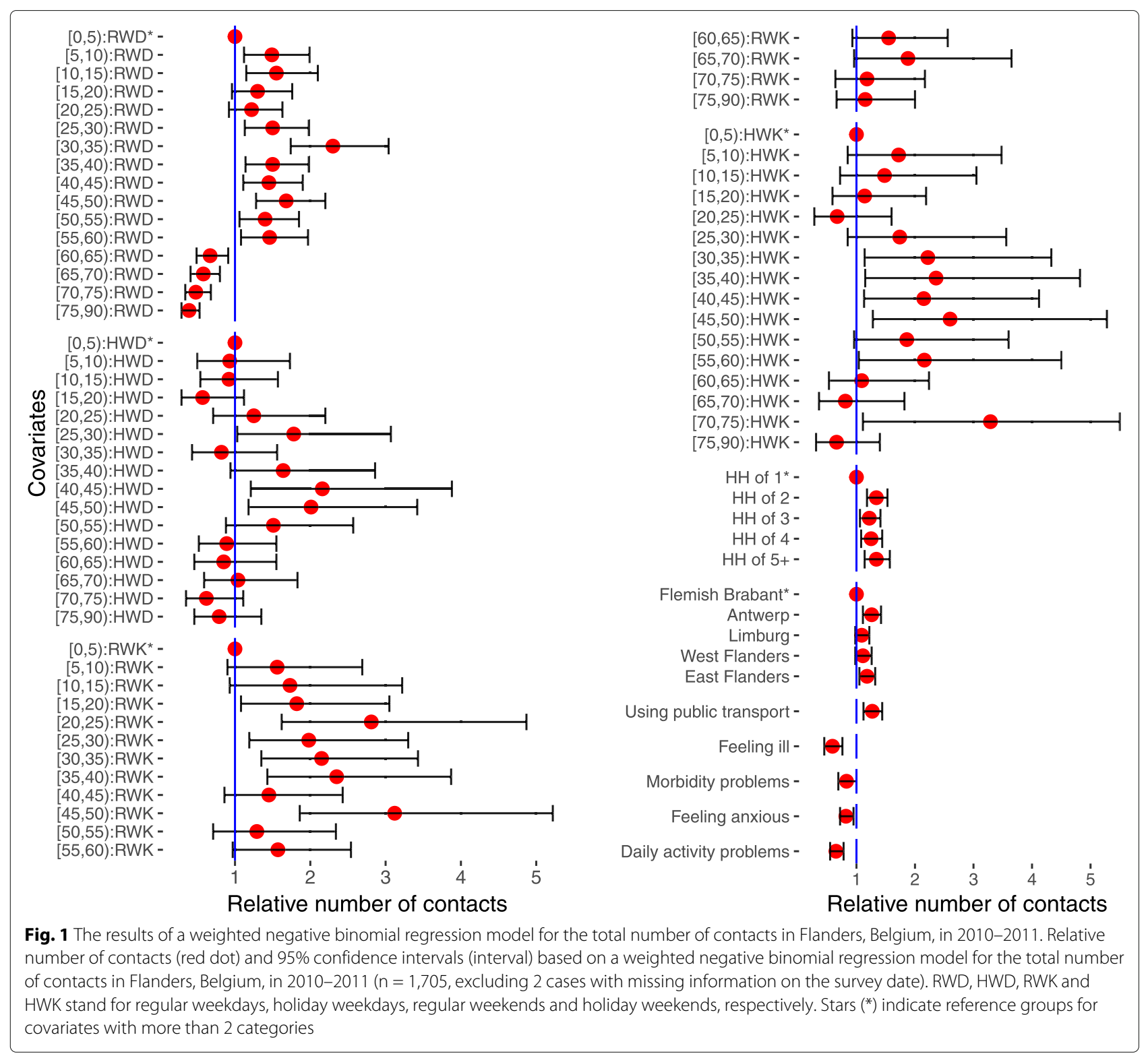

\section{People living in an elderly/nursing home}

Forty-six people reported living in a nursing/elderly home, with ages ranging from 79 to 99 years. Most of them had health problems: some problems or not being able to perform their daily activities (96\%), some problems walking $(67 \%)$ or staying in bed all the time $(15 \%)$; some problems with self-care (46\%) or not being able to care for themselves (43\%); and experiencing mild to serious pain (85\%) and anxiety (48\%). These people reported 13.7 contacts on average, significantly higher than those aged $60+$ years and living at home $(\mathrm{P}<0.0 .001$, Mann-Whitney test). No statistically significant difference in the number of contacts for people in elderly/nursing homes was found with respect to the residence size $(\mathrm{P}=0.47$, Kruskal-Wallis test for 3 groups of residence sizes: $<50,50-100$ and
$100+)$. We compared people living in an elderly/nursing home with people aged $60+$ years living at home with respect to their social interaction with other age groups (see Additional file 3 Figure S2): almost no interaction with young children and teenagers was observed for people living in an elderly/nursing home, while this interaction was more observed for people aged $60+$ years living at home.

\section{Overall contact patterns}

The contact patterns by age group were summarized in a contact matrix displaying ages from 0 to 90 years, whose elements represent the contact rate between an individual in a given age group and an individual in another age group in the population of Flanders. The resulting contact 
a)

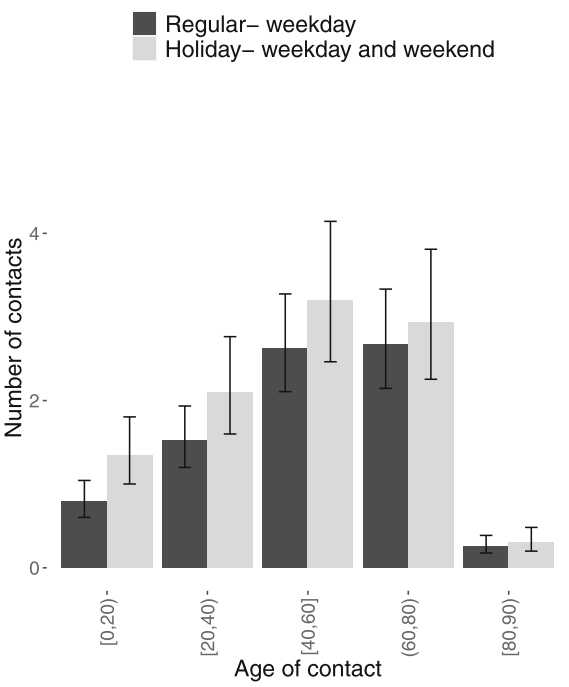

b)

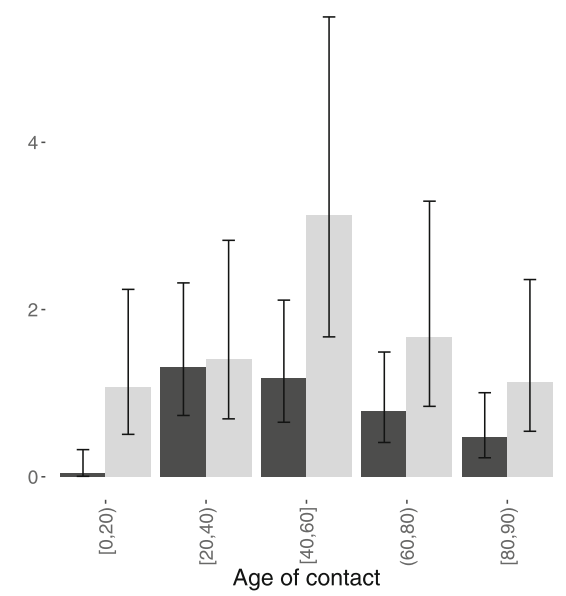

Fig. 2 Age-specific number of contacts of older people for the 2010-2011 survey. a Age-specific number of contacts of people aged 61-79 years. b Age-specific number of contacts of people aged $80+$ years

matrix shown in Fig. 3 is described by the pronounced main diagonal indicating contacts with individuals in the same age group, e.g., at home, at school and at work, and the 2 less-pronounced sub-diagonals representing contacts between generations, e.g., children and their parents.

\section{Sex differences in mixing patterns}

Figure 4 shows the age- and sex-specific average number of contacts. The assortative mixing pattern characterized by the main diagonal was still observed for all interactions. The measures of assortativeness for all ages provided no differences for same sex vs. different sexes, with overlapping $95 \%$ BCI of $I_{s}^{2}$ indices $(0.39[0.35 ; 0.45]$ and 0.42 [0.40;0.45] vs 0.48 [0.43;0.54] and 0.44 [0.42;0.47], for male-male and female-female vs. male-female and femalemale, respectively). Focusing on people aged less than 30 years, we noticed that the assortative mixing pattern was more pronounced in male-to-male and female-to-female

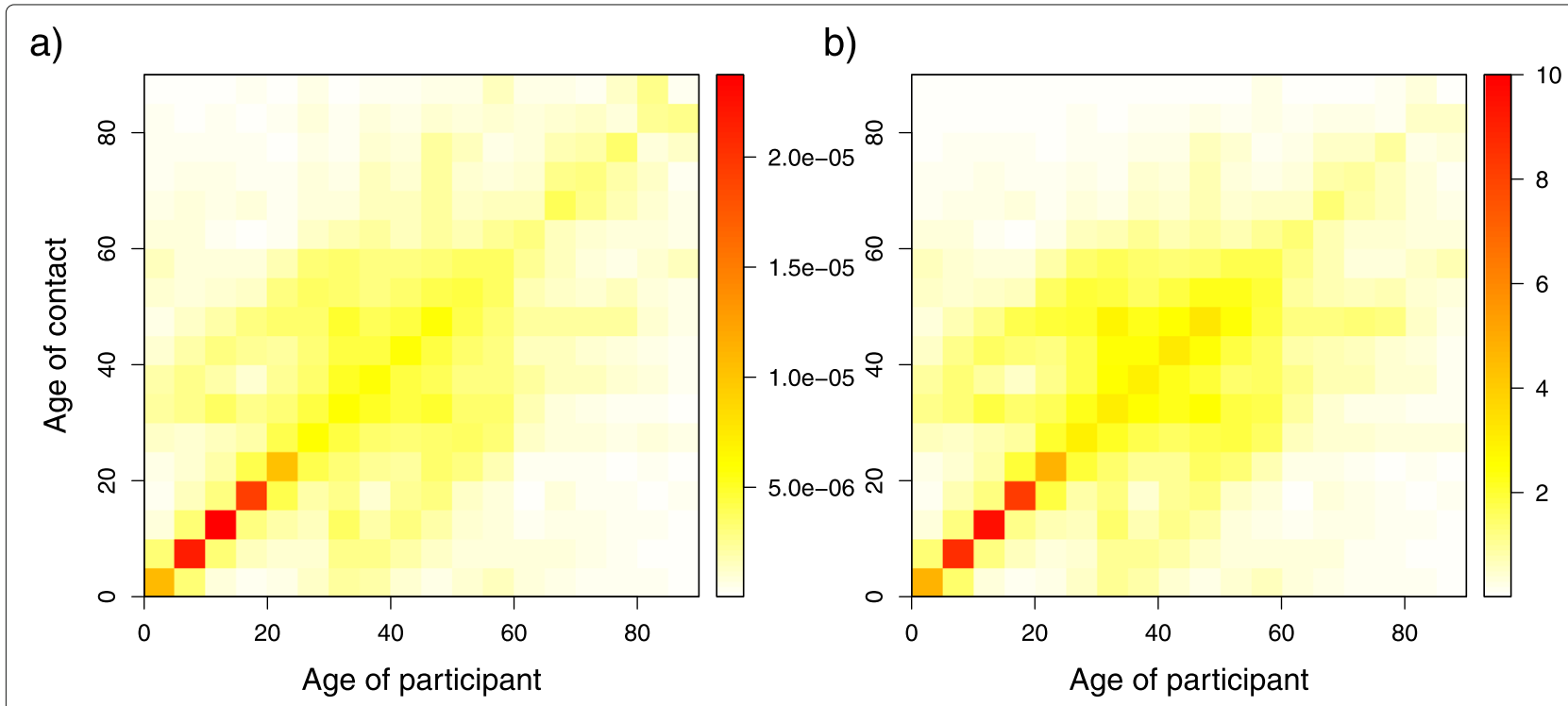

Fig. 3 Estimated age-specific contact rates and average number of contacts for the 2010-2011 survey. a Estimated symmetric age-specific contact rates. b Age-specific average number of contacts. The color scale indicates the contact rates from low (white) to high (red) 
a)

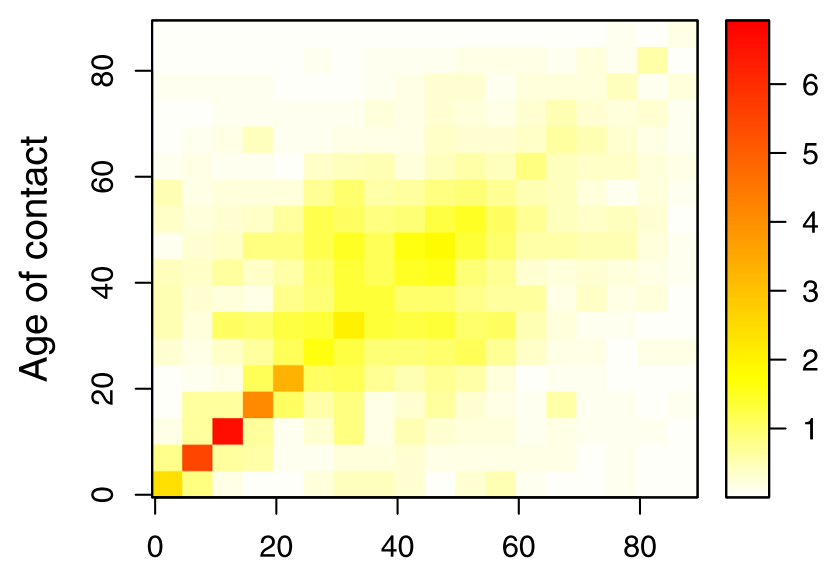

c)

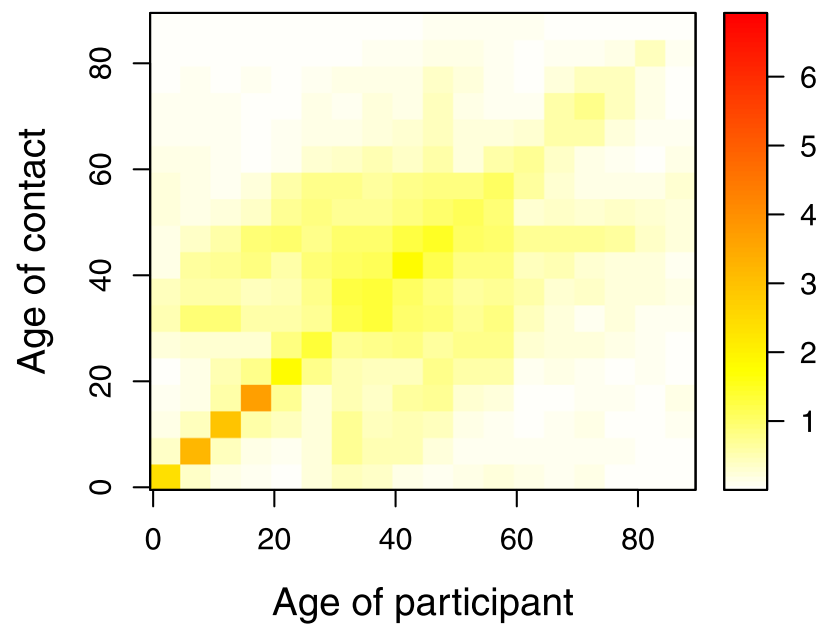

b)

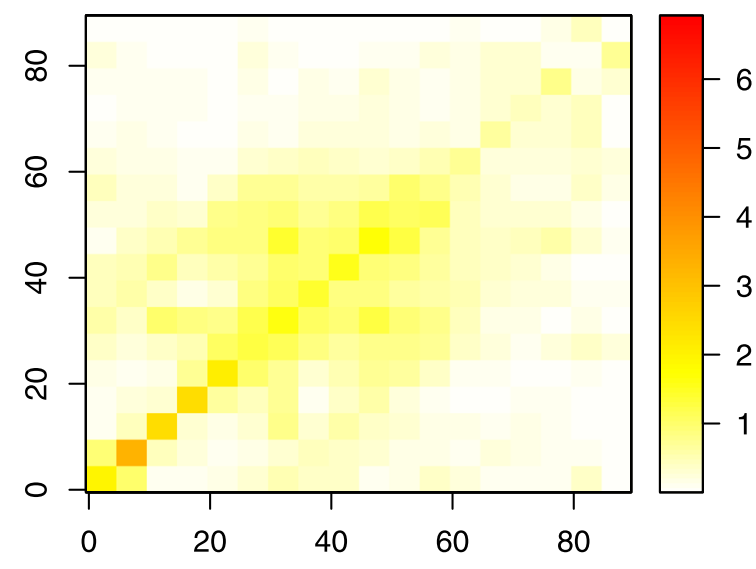

d)

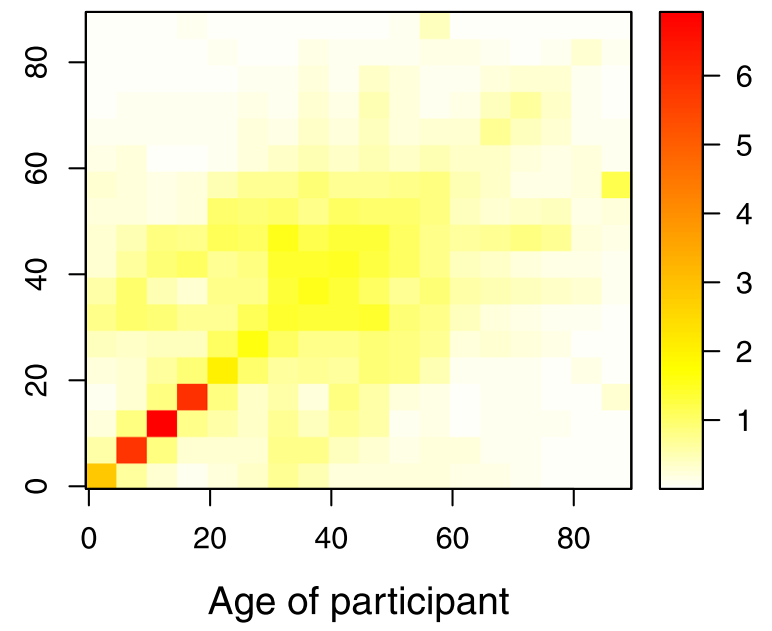

Fig. 4 Estimated age- and sex-specific average number of contacts for the 2010-2011 survey. a male-male contacts, b male-female contacts, $\mathbf{c}$ female-male contacts and $\mathbf{d}$ female-female contacts. The color scale indicates the number of contacts from low (white) to high (red)

contacts. Specifically, for contacts made between people in the same age groups, the average number of maleto-male contacts and female-to-female contacts were 3.9 (BCI $[3.5 ; 5.0])$ and $4.2(\mathrm{BCI}[3.7 ; 4.9])$, respectively. These figures reduced to 2.3 (BCI $[2.0 ; 2.8]$ ) for male-tofemale contacts and 2.6 (BCI $[2.3 ; 3.1])$ for female-to-male contacts. The inter-generational mixing pattern (mostly parent-child), marked by the two sub-diagonals, was similar for females and males. The relative incidences for both sexes also followed a similar pattern, with peaks at approximately 15 years of age and between 40 and 45 years of age, and no difference was found in the overall RI of males compared to that of females (see Figure S3 in Additional file 3).

\section{School closure impact}

We observed a significant difference in $R_{0}$ between holiday and regular periods: the relative change in $R_{0}$ was 0.71 (BCI [0.60; 0.85]), or equivalently a $29 \%$ reduction in $R_{0}$ for the holiday vs. the regular period. When comparing the relative change in $R_{0}$ from a weekday to the weekend, a slightly higher reduction of $30 \%$ was observed. The difference in RI by age group is shown in Fig. 5. The comparison of weekdays to weekends showed that the RI decreased significantly in the age group 0-15 years, while it was higher in the age group $[60,65)$ and $[70,75)$ on the weekend compared with the weekday. The RI also decreased from regular to holiday periods for the 3 age groups from 5 to 20 years, with the highest reduction observed in the age 
a)

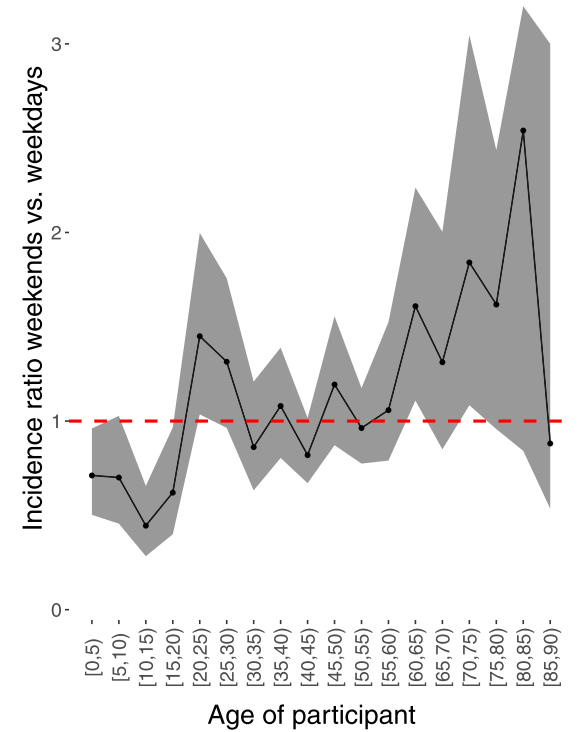

b)

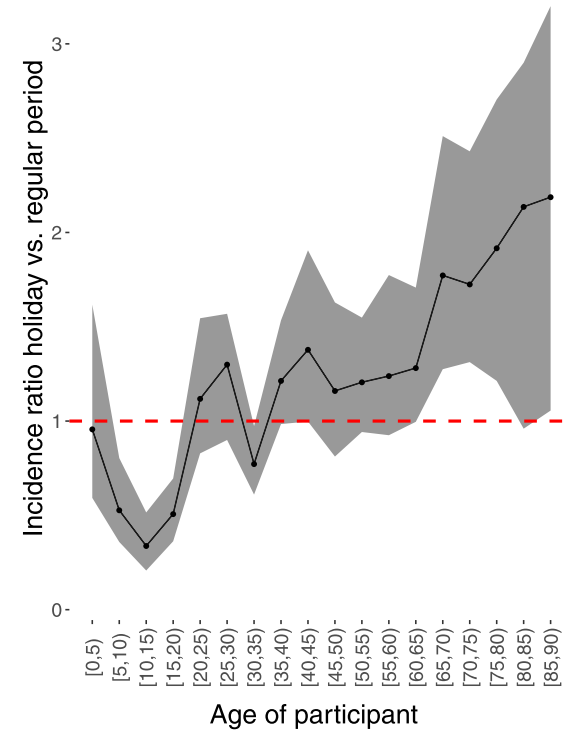

Fig. 5 Ratio of relative incidences. a Ratio of relative incidences between weekends and weekdays. b Ratio of relative incidences between holidays and regular periods for the 2010-2011 survey. Shaded areas indicate 95\% percentile bootstrap confidence intervals

group 10-15 years. When comparing regular to holiday periods, we observed an increase in the RI for participants aged 65 to 80 years, though the RI variability for this age group was considerably high.

\section{Comparing the contact surveys in 2006 and 2010-2011}

The age distribution of the population of Flanders by age group did not change much in almost 5 years (Additional file 3 Figure S4a). The distribution of participants in the survey 2010-2011 was closer to the population of Flanders than that in the 2006 survey, especially for people older than 60 years. Participants aged between $0-20$ years were over-sampled, while age groups 30-45 and 65-85 were under-sampled in the 2006 survey. The average number of contacts by age, adjusted for age, household size and types of days (weekdays/weekend days), in the two surveys is presented in Additional file 3 Figure S4b. The largest difference in the number of survey participants was found for the age group 70-75.

\section{Degree distribution from aggregated data}

The result of the random forest analysis is shown in Additional file 3 Figure S5: sex yielded the lowest mean decrease in accuracy, so it was removed. Significant predictors for both mean and over-dispersion parameters were further selected using the likelihood ratio test. Accordingly, the mean and over-dispersion regressions in the final model included household size and all interaction effects of three variables: age groups, microscopic time and macroscopic time.
The results of the GAMLSS model for the total number of contacts using the aggregated data from both surveys are shown in Additional file 3 Table S4. After controlling for other factors, the household size was still significant, with a higher number of contacts recorded by participants living in a larger household. On regular weekdays, participants aged 60+ years reported the lowest number of contacts in both the 2006 and 2010-2011 surveys. During the same period, participants aged $45-50$ years had the highest number of contacts in the 2006 survey, while participants aged $30-35$ years had the highest number of contacts in the 2010-2011 survey. We observed a significant effect of microscopic time settings: in particular, participants aged 0-5 years reported a significantly lower number of contacts during weekends than regular weekdays, with RNC being 0.33 (CI [0.24; 0.45]) for the 2006 survey, while no significant differences in the number of contacts between during the regular weekday and the holiday weekdays was found for this age group. The main effect of macroscopic time (calendar year) and its interaction effects with age group and microscopic time settings were significant. On regular weekdays, participants aged 0-5 years old in the 2010-2011 survey reported a lower number of contacts than those in the 2006 survey: RNC is 0.64 (CI[0.46; 0.89]).

\section{Comparing the contact matrices for 2006 and 2010-2011}

The difference between contact matrices estimated from the 2006 and 2010-2011 social contact surveys was negligible (Fig. 6). Similar age and parent-child mixing patterns 


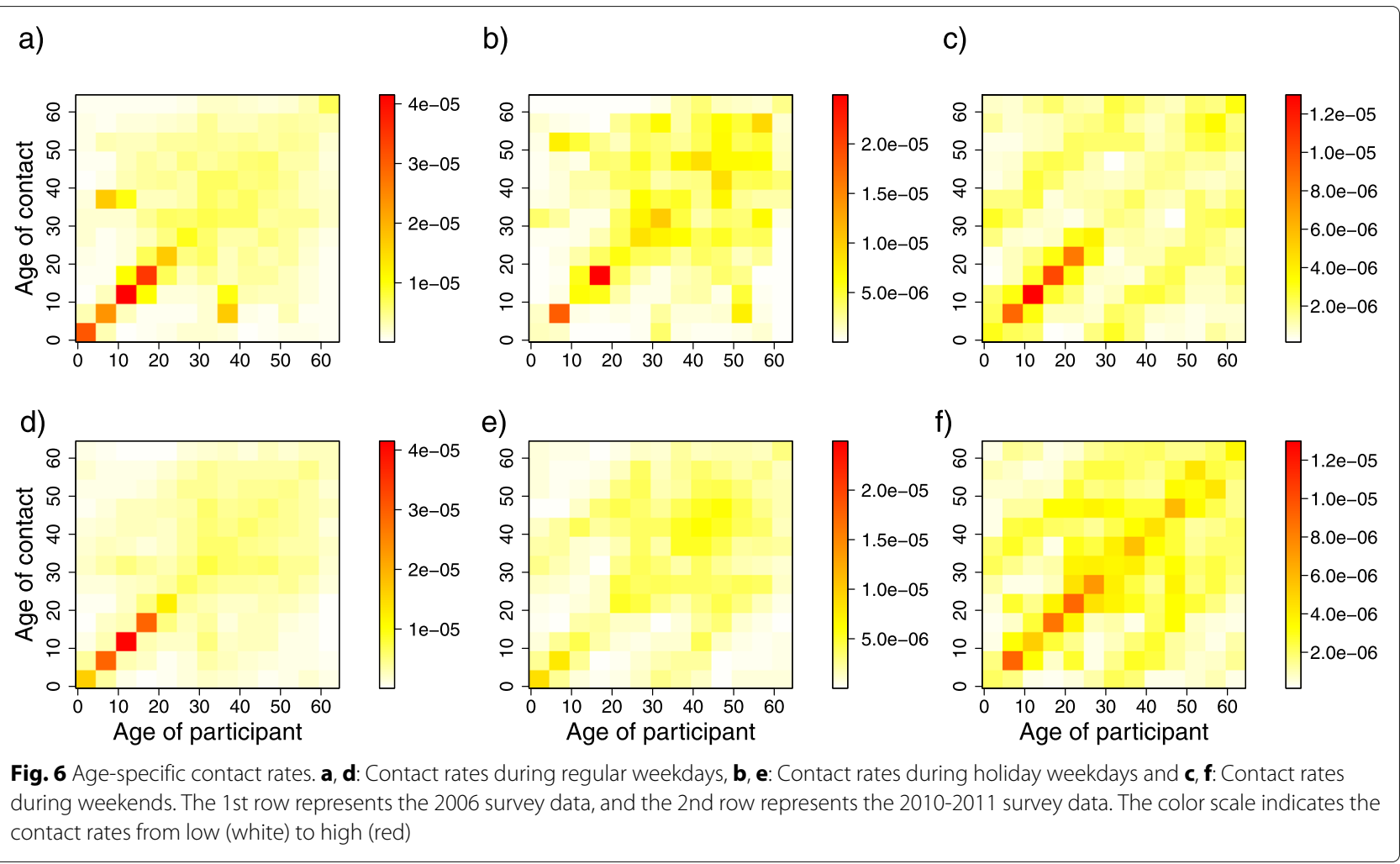

were observed for all contact matrices. The degree of assortativeness measured by $\mathrm{Q}$ and $I^{2}$ indices (Table 2) were comparable in each microscopic time period, as evidenced by the overlapping BCIs. The relative incidences in 2006 and 2010-2011 (Additional file 3 Figure S6) were also similar in both regular weekdays and holiday weekdays but were moderately dissimilar on the weekend, as the highest relative incidence was found in two different age classes (10-15 years and 20-25 years for 2006 and 2010-2011, respectively).

The relative change in $R_{0}$, RRI and the ratio of transmission rates were used to further compare the epidemiological differences between contact matrices from the two surveys. The relative changes in $R_{0}$ are presented in Fig. $7 \mathrm{a}$. Based on the $95 \% \mathrm{BCI}$ of relative changes in $R_{0}$, significant changes between contact matrices of 2006 (numerator) and 2010-2011 (denominator) were only observed during the weekend, with an upper bound of the $\mathrm{BCI}$ close to 1 . Figure $7 \mathrm{~b}$ presents the changes in relative incidence between the 2006 and 2010-2011 social contact matrices stratified over 13 age groups and 3 microscopic time settings. Similar to the results of the relative change in $R_{0}$, there was no evidence to support changes in RI over time, except for the age group 10-15 years during the weekend, where the RI was significantly higher in 2006 than in 2010-2011. The comparison of contact matrices based on the ratio of cell-wise contact rates in each microscopic time setting is provided in Additional file 3 Figures S7, S8 and S9, for which the BCIs included a correction for multiple testings. We only found few significant differences in contact rates during holiday weekdays between 2006 and 2010-2011, mostly for participants aged $50+$ years.

We compared the fit of the two contact matrices in 2006 and 2010-2011 to VZV serological data (see Additional file 3 Figures S10). Although the contact matrix

Table 2 Assortativeness measures

\begin{tabular}{|c|c|c|c|c|}
\hline \multirow[t]{2}{*}{ Microscopic time } & \multicolumn{2}{|l|}{$Q$ index } & \multicolumn{2}{|l|}{$I_{s}^{2}$ index } \\
\hline & Survey 2006 & Survey 2010-2011 & Survey 2006 & Survey 2010-2011 \\
\hline Regular weekday & $0.21[0.15 ; 0.25]$ & $0.21[0.18 ; 0.23]$ & $0.53[0.43 ; 0.68]$ & $0.50[0.45 ; 0.59]$ \\
\hline Regular holiday & $0.15[0.10 ; 0.19]$ & $0.11[0.07 ; 0.15]$ & $0.54[0.46 ; 0.64]$ & $0.58[0.49 ; 0.68]$ \\
\hline Weekend & $0.14[0.10 ; 0.18]$ & $0.12[0.09 ; 0.15]$ & $0.75[0.64 ; 0.93]$ & $0.61[0.54 ; 0.67]$ \\
\hline
\end{tabular}



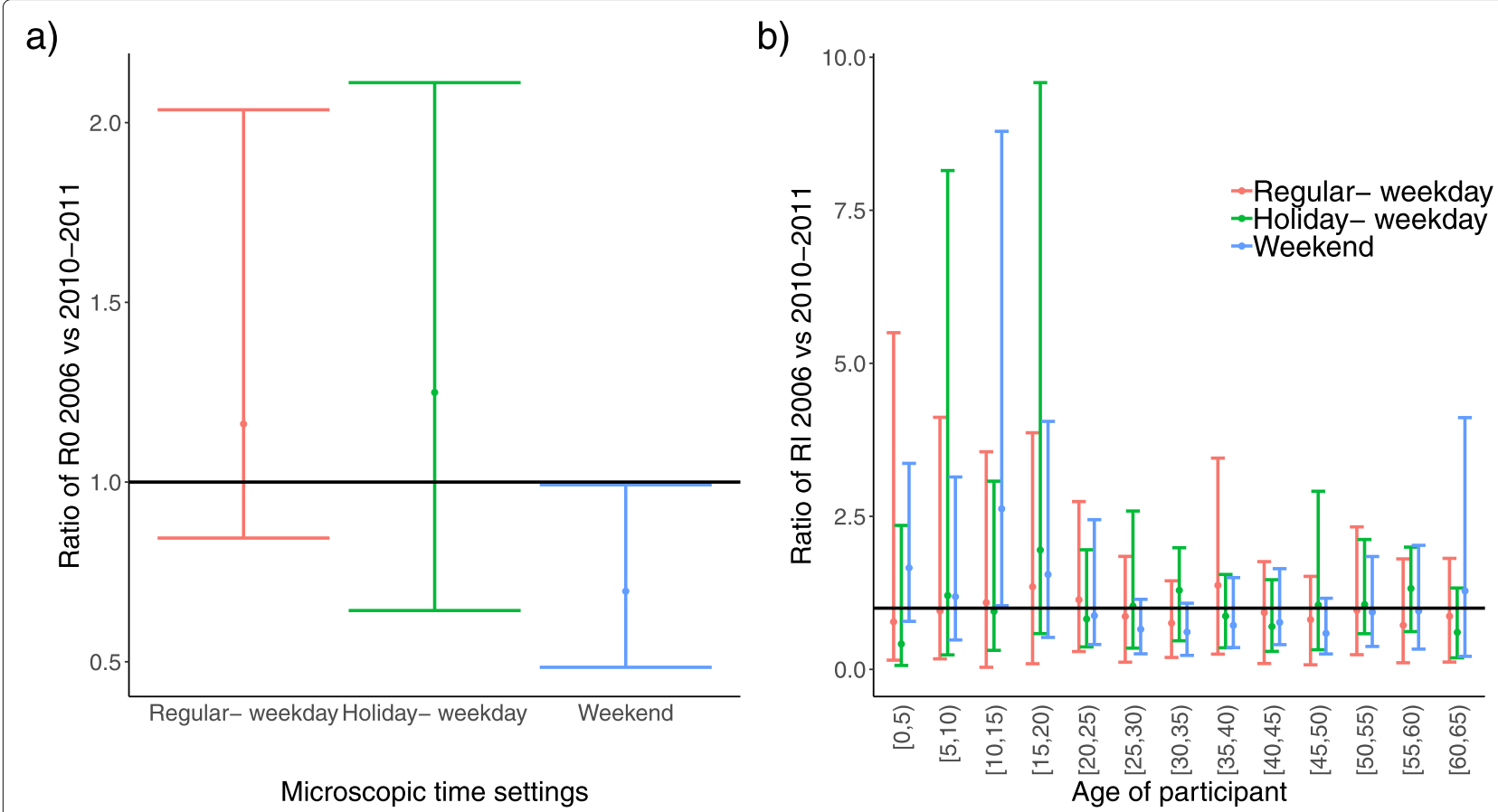

Fig. 7 The ratio of basic reproduction numbers and of relative incidence by 5 -year age groups. $\mathbf{a}$ The ratio of basic reproduction numbers and $\mathbf{b}$ the ratio of relative incidence by 5-year age groups for the 2006 and 2010-2011 social contact surveys in Flanders. The estimated relative changes in $R_{0}$ and RRIs are shown by the dots with $95 \% \mathrm{BCl}$ for each microscopic time setting. BCls are obtained after Bonferroni correction with an overall significance level of 0.05 . The horizontal line at 1 means no difference

in 2006 produced a slightly better fit than the contact matrix in 2010-2011 (AIC: 1379 vs 1383), the observed prevalence and force of infection by age group between the two surveys almost completely overlapped. We found no significant difference in $R_{0}$ obtained from the contact matrices in 2006 and in 2010-2011 (10.7 BCI[6.23; 16.45] vs. 7.8 BCI [5.46; 7.51], respectively).

\section{Discussion}

Social contact surveys provide empirical data on populations' mixing patterns that can inform mathematical models of infectious diseases. In Belgium, two large diary-based social contact surveys were conducted in 2006 and 2010-2011. In this work, we present the results of the latest survey, discussing the impact of microscopic time differences on mixing patterns and comparing the 2010-2011 data with the 2006 data (in Flanders only). This approach allowed for assessing changes over macroscopic time differences, albeit in the limiting scenario of two surveys conducted only 4-5 years apart in a region where demography has remained fairly stable.

The association rules revealed that contacts of less than $15 \mathrm{~min}$ with non-household members usually do not involve skin-to-skin touching. This finding is in line with the results of the 2006 survey [28]. To investigate the contact profiles of participants, we performed clustering analysis. Our clustering results are comparable to the results in [3], in which a two-step clustering approach was applied to contact data from eight European countries. Specifically, we endorsed the "school profile", "professional profile", and "leisure profile" from [3], with more contacts during leisure activities during weekends.

Demographic factors, including age, household and province of residence, had significant effects on the number of contacts, as did the temporal factors, e.g., weekdays vs weekend days or regular terms vs holiday periods $[9,10,16,22,40]$. It is noted that the interaction between people aged $60+$ years and young children/teenagers was significantly higher during holidays and weekends compared to regular- weekdays. For people living in an elderly/nursing home, however, almost no contacts with young children/teenagers were reported. Using public transportation was associated with a higher number of contacts in total. Our analysis also showed that those who reported to feel ill had fewer contacts than those who reported to be healthy $[6,10,15-17]$. This also holds for participants reporting health problems such as anxiety or those experiencing problems in daily activities.

There was evidence, at least among school-aged children, that contact patterns were assortative with respect to both age and sex. While an assortative mixing pattern 
with respect to age was still observed in adults, albeit with lower contact rates, an assortative mixing pattern with respect to sex disappears in people aged $30+$ years. This analysis was also performed in [41], where a hierarchical Bayesian model was used to infer age-specific contact rates between sexes. In contrast to [41], we did not find significant differences in infection risk between males and females. There are some reasons that may explain this difference. First, we aggregated the age of participants in 20 age classes instead of using continuous age, which can incur an inevitable loss of detail. Second, the dispersion parameter in our model was assumed to be agedependent, while it was treated as a nuisance parameter in [41] to avoid computation challenges. In addition, we used diary weights in contact modelling to account for under/over-sampling over the age of participants, while weights were not taken into account in the model of [41].

We found that the number of contacts was lower on weekends than on weekdays and during holidays compared to regular periods. We find a 30\% (BCI:[17; 37\%) reduction in $R_{0}$ for weekends versus weekdays or a $29 \%$ (BCI:[14; 40\%]) reduction in $R_{0}$ for holidays versus regular periods. This result is consistent with the results of other studies $[8,19,40,42,43]$. However, computing the age-specific relative incidence showed that this reduction was due to the younger age classes, both during weekends and during holidays. Additionally, the age-specific relative incidence showed that during holidays, there was a more complex change than during regular weekends: while younger people had a lower relative incidence, people older than 60 years had an increased relative incidence during holidays.

Contact matrices were compared in different microscopic time settings, namely, regular weekdays, holiday weekdays and weekends, to obtain insights into possible changes in contact patterns between 2006 and 2010-2011 (Fig. 6). Our result showed that irrespective of microscopic time settings, the contact patterns in 2006 and 2010-2011 followed the same trend of assortativeness. Furthermore, we observed pronounced inter-generational age mixing (the two sub-diagonals of the contact matrices), most likely indicating parent-child mixing patterns. This finding supports the evidence that households are central units in the epidemiology of airborne infections, e.g., influenza and SARS, because of the nature of the frequent and intimate contacts among household members. Children thus can have a bridging function, allowing for the spread of infection within households and to other households, from schools to workplaces or vice versa in a community [44]. The mixing patterns obtained from the contact matrices in 2006 and 2010-2011 in our study are in agreement with mixing patterns observed in similar studies $[9,10]$. The relative incidences based on the 2006 and 2010-2011 data were quite similar between regular weekdays and holiday weekdays but are dissimilar on the weekend, as the highest relative incidence was found in two different age classes (10-15 years and 20-25 years for 2006 and 2010-2011, respectively).

In this study, we found that contact patterns remained fairly constant over 4-5 years. Additionally, within each microscopic time period, no substantial changes in the spread of infection, measured by the relative basic reproduction number and age-specific incidences, were observed (Fig. 7). After taking into account multiple testings, the pair-wise comparison of contact rates over time presented only few significant differences during holiday weekdays, mostly for people aged $50+$ years. While the comparison of only two observational periods about five years apart can be considered a limitation, to the best of our knowledge, this is the first study, that investigates empirically whether contact rates remain stable, in the absence of major shocks to risk perception (as we expect to observe in the SARS-CoV-2 pandemic emergence year 2020) and demography.

\section{Conclusions}

The second social contact survey in Flanders, Belgium, endorses the findings of its 2006 predecessor and adds important information on the social mixing patterns of people older than 60 years of age. Based on this analysis, the mixing patterns of people older than 60 years exhibit considerable heterogeneity. From the comparison of the two surveys, we suggest that social contact rates can be assumed stable in Flanders over a time span of 5 years when no major shocks to risk perceptions or demography occur.

\section{Abbreviations \\ BCls: Bootstrap confidence intervals; GAMLSS: Generalised additive models for location scale and shape; LRT: Likelihood ratio test; $R_{0}$ : Basic reproduction number; RI: Relative incidence; RNC: Relative number of contacts; RRI: Ratio of relative incidences; VZV: Varicella zoster virus}

\section{Supplementary Information}

The online version contains supplementary material available at https://doi.org/10.1186/s12879-021-05949-4.

Additional file 1: Descriptive data analysis of the 2010-2011 dataset

Additional file 2: Data mining techniques

Additional file 3: Supplementary results of the 2006 and 2010-2011 social contact survey data analyses

Acknowledgements

Not applicable.

Authors' contributions

$\mathrm{NH}$ and PB designed and coordinated the survey. $\mathrm{NH}$ conceived the study and laid out a paper structure. LW and KVK performed data cleaning, TVH and YWK conducted the data analyses and drafted the manuscript in consultation with all the other authors. NH, PC, KVK and SV made substantial revisions to the manuscript. All authors contributed to the final version of the manuscript. All authors read and approved the final manuscript. 


\section{Funding}

This work received funding from the European Research Council (ERC) under the European Union's Horizon 2020 research and innovation programme (grant agreement 682540 - TransMID). LW gratefully acknowledge support from the Fonds voor Wetenschappelijk Onderzoek (FWO, postdoctoral fellowship 1234620N)

\section{Availability of data and materials}

The datasets used and analysed during the current study are available via www.socialcontactdata.org within the social contact data sharing initiative which is part of the ERC consolidator grant "TransMID".

\section{Declarations}

\section{Ethics approval and consent to participate}

No physical samples were collected as part of this study. A verbal consent was given prior to participation during the recruitment over the phone. People who agreed verbally to participate were then sent a written questionnaire and diary. They were able to refuse participation even after verbal agreement by not filling in the questionnaire and diary, and/or by not sending it back by postal services. Thus, the fact that they filled in the questionnaire and diary and chose to send it in functions as a written consent. We obtained similar verbal consent with implicit written confirmation from the next of kin, caretakers or guardians on behalf of dependent participants (e.g. children). All records of participants were anonymized before its use.

Both datasets used for the current study are shared within the social contact data sharing initiative which is part of the ERC consolidator grant "TransMID" which received ethical approval from the Hasselt University Medical Ethical Committee (CME2016/618)

\section{Consent for publication}

Not applicable.

\section{Competing interests}

The authors declare that they have no competing interests.

\section{Author details}

${ }^{1}$ I-Biostat, Data Science Institute, Hasselt University, Martelarenlaan 42, 3500 Hasselt, Belgium. ${ }^{2}$ The Janssen Pharmaceutical Companies of Johnson \& Johnson, Antwerpen, Belgium. ${ }^{3}$ Centre for Health Economic Research and Modelling Infectious Diseases, Vaccine \& Infectious Diseases Institute, University of Antwerp, Universiteitsplein 1, 2610 Antwerp, Belgium. ${ }^{4}$ School of Public health and Community Medicine, University of New South Wales, 2052 Sydney, Australia.

\section{Received: 1 October 2020 Accepted: 2 March 2021}

Published online: 18 March 2021

\section{References}

1. Anderson RM, May RM. Infectious Diseases of Humans: Dynamics and Control. Great Clarendon: Oxford University Press; 1992.

2. Wallinga J, Teunis P, Kretzschmar M. Using data on social contacts to estimate age-specific transmission parameters for respiratory-spread infectious agents. Am J Epidemiol. 2006;164(10):936-44.

3. Kretzschmar M, Mikolajczyk RT. Contact profiles in eight European countries and implications for modelling the spread of airborne infectious diseases. PLoS ONE. 2009;4(6):5931.

4. Goeyvaerts N, Hens N, Ogunjimi B, Aerts M, Shkedy Z, Damme PV, Beutels $P$. Estimating infectious disease parameters from data on social contacts and serological status. J R Stat Soc: Ser C: Appl Stat. 2010;59(2): 255-77.

5. Willem L, Van Kerckhove K, Chao DL, Hens N, Beutels P. A nice day for an infection? Weather conditions and social contact patterns relevant to influenza transmission. PLoS ONE. 2012;7(11):48695

6. Van Kerckhove K, Hens N, Edmunds WJ, Eames KT. The impact of illness on social networks: implications for transmission and control of influenza. Am J Epidemiol. 2013;178(11):1655-62.
7. Ewing A, Lee EC, Viboud C, Bansal S. Contact, travel, and transmission: The impact of winter holidays on Influenza dynamics in the United States. J Infect Dis. 2017;215(5):732-9.

8. De Luca G, Van Kerckhove K, Coletti P, Poletto C, Bossuyt N, Hens N, Colizza $\vee$. The impact of regular school closure on seasonal influenza epidemics: a data-driven spatial transmission model for Belgium. BMC Infect Dis. 2018;18(1):29.

9. Mossong J, Hens N, Jit M, Beutels P, Auranen K, Mikolajczyk R, Massari M, Salmaso S, Tomba GS, Wallinga J, et al. Social contacts and mixing patterns relevant to the spread of infectious diseases. PLoS Med. 2008;5(3):74.

10. Hoang T, Coletti P, Melegaro A, Wallinga J, Grijalva CG, Edmunds JW, Beutels P, Hens N. A systematic review of social contact surveys to inform transmission models of close-contact infections. Epidemiology (Cambridge, Mass.) 2019;30(5):723-36.

11. Horton KC, Hoey AL, Béraud G, Corbett EL, White RG. Systematic review and meta-analysis of sex differences in social contact patterns and implications for tuberculosis transmission and control. Emerg Infect Dis. 2020;26(5):910.

12. Ogunjimi B, Hens N, Goeyvaerts N, Aerts M, Van Damme P, Beutels P. Using empirical social contact data to model person to person infectious disease transmission: an illustration for varicella. Math Biosci. 2009;218(2): 80-7.

13. Held L, Hens N, D O'Neill P, Wallinga J. Handbook of Infectious Disease Data Analysis. US: CRC Press; 2019.

14. Willem L, Hoang TV, Funk S, Coletti P, Beutels P, Hens N. SOCRATES: an online tool leveraging a social contact data sharing initiative to assess mitigation strategies for COVID-19. BMC Res Notes. 2020;13(1):1-8. https://doi.org/10.1186/s13104-020-05136-9.

15. DeStefano F, Haber M, Currivan D, Farris T, Burrus B, Stone-Wiggins B, McCalla A, Guled H, Shih H, Edelson P, et al. Factors associated with social contacts in four communities during the 2007-2008 influenza season. Epidemiol Infect. 2011;139(8):1181-90.

16. Chen S-C, You S-H, Ling M-P, Chio C-P, Liao C-M. Use of seasonal influenza virus titer and respiratory symptom score to estimate effective human contact rates. J Epidemiol. 2012;22(4):353-63.

17. Stein ML, van der Heijden PG, Buskens $V$, van Steenbergen JE, Bengtsson L, Koppeschaar CE, Thorson A, Kretzschmar ME. Tracking social contact networks with online respondent-driven detection: who recruits whom? BMC Infect Dis. 2015;15(1):522.

18. Mikolajczyk R, Akmatov M, Rastin S, Kretzschmar M. Social contacts of school children and the transmission of respiratory-spread pathogens. Epidemiol Infect. 2008;136(6):813-22.

19. Hens N, Ayele GM, Goeyvaerts N, Aerts M, Mossong J, Edmunds JW, Beutels $P$. Estimating the impact of school closure on social mixing behaviour and the transmission of close contact infections in eight European countries. BMC Infect Dis. 2009;9(1):1-12.

20. Horby P, Thai PQ, Hens N, Yen NTT, Thoang DD, Linh NM, Huong NT, Alexander N, Edmunds WJ, Duong TN, et al. Social contact patterns in Vietnam and implications for the control of infectious diseases. PloS ONE. 2011;6(2):16965.

21. Johnstone-Robertson SP, Mark D, Morrow C, Middelkoop K, Chiswell M, Aquino LD, Bekker L-G, Wood R. Social mixing patterns within a South African township community: implications for respiratory disease transmission and control. Am J Epidemiol. 2011;174(11):1246-55.

22. Béraud G, Kazmercziak S, Beutels P, Levy-Bruhl D, Lenne X, Mielcarek N, Yazdanpanah Y, Boëlle P-Y, Hens N, Dervaux B. The French connection: The first large population-based contact survey in France relevant for the spread of infectious diseases. PLoS ONE. 2015;10(7):0133203.

23. Dodd PJ, Looker C, Plumb ID, Bond V, Schaap A, Shanaube K, Muyoyeta M, Vynnycky E, Godfrey-Faussett P, Corbett EL, et al. Age-and sex-specific social contact patterns and incidence of mycobacterium tuberculosis infection. Am J Epidemiol. 2015;183(2):156-66.

24. Santermans E, Van Kerckhove K, Azmon A, Edmunds WJ, Beutels P, Faes C, Hens N. Structural differences in mixing behavior informing the role of asymptomatic infection and testing symptom heritability. Math Biosci. 2017;285:43-54.

25. Prem K, Cook AR, Jit M. Projecting social contact matrices in 152 countries using contact surveys and demographic data. PLoS Comput Biol. 2017;13(9):1-21. 
26. Arregui S, Iglesias MJ, Samper S, Marinova D, Martin C, Sanz J, Moreno Y. Data-driven model for the assessment of mycobacterium tuberculosis transmission in evolving demographic structures. Proc Natl Acad Sci. 2018;115(14):3238-45.

27. Arregui S, Aleta A, Sanz J, Moreno Y. Projecting social contact matrices to different demographic structures. PLoS Comput Biol. 2018;14(12): 1006638.

28. Hens N, Goeyvaerts N, Aerts M, Shkedy Z, Van Damme P, Beutels P. Mining social mixing patterns for infectious disease models based on a two-day population survey in Belgium. BMC Infect Dis. 2009;9(1):1-8.

29. Kifle YW, Goeyvaerts N, Van Kerckhove K, Willem L, Kucharski A, Faes C, Leirs $\mathrm{H}$, Hens N, Beutels P. Animal ownership and touching enrich the context of social contacts relevant to the spread of human infectious diseases. PloS ONE. 2016;11(2):e0148718.

30. TransMID: Socical Contact Data. 2017. http://www.socialcontactdata. org/. Accessed 3 May 2018.

31. Breiman L. Random forests. Mach Learn. 2001;45(1):5-32.

32. Maechler M, Rousseeuw P, Struyf A, Hubert M, Hornik K. cluster: Cluster Analysis Basics and Extensions. 2021. https://CRAN.R-project.org/ package $=$ cluster, $\mathrm{R}$ package version 2.1.1.

33. Maaten LVD, Hinton G. Visualizing data using t-SNE. J Mach Learn Res. 2008;9:2579-605.

34. Rigby RA, Stasinopoulos DM. Generalized additive models for location, scale and shape, (with discussion). Applied Statistics. 2005;54(3):507-54.

35. Hens N, Wallinga J. Design and analysis of social contact surveys relevant for the spread of infectious diseases. Wiley StatsRef: Statistics Reference Online. 2019;39-57.

36. Efron B, Tibshirani RJ. An Introduction to the Bootstrap. US: CRC press; 1994.

37. Gupta S, Anderson RM, May RM. Networks of sexual contacts: implications for the pattern of spread of HIV. AIDS (London, England). 1989;3(12):807-17.

38. Farrington C, Whitaker H, Wallinga J, Manfredi P. Measures of disassortativeness and their application to directly transmitted infections. Biom J: J Math Meth Biosci. 2009;51(3):387-407.

39. Diekmann O, Heesterbeek JAP, Metz JA. On the definition and the computation of the basic reproduction ratio $R_{0}$ in models for infectious diseases in heterogeneous populations. J Math Biol. 1990;28(4):365-82.

40. Chen S-C, You Z-S. Social contact patterns of school-age children in taiwan: comparison of the term time and holiday periods. Epidemiol Infect. 2015;143(6):1139-47.

41. van de Kassteele J, van Eijkeren J, Wallinga J. Efficient estimation of age-specific social contact rates between men and women. Ann Appl Stat. 2017;11(1):320-39.

42. Jackson C, Mangtani P, Vynnycky E, Fielding K, Kitching A, Mohamed H, Roche A, Maguire H. School closures and student contact patterns. Emerg Infect Dis. 2011;17(2):245.

43. Modchang C, lamsirithaworn S, Auewarakul P, Triampo W. A modeling study of school closure to reduce influenza transmission: A case study of an influenza $A(\mathrm{H} 1 \mathrm{~N} 1)$ outbreak in a private thai school. Math Comput Model. 2012;55(3-4):1021-33.

44. Goeyvaerts N, Santermans E, Potter G, Torneri A, Van Kerckhove K, Willem $L$, Aerts M, Beutels P, Hens N. Household members do not contact each other at random: implications for infectious disease modelling. Proc R Soc B. 2018;285(1893):20182201.

\section{Publisher's Note}

Springer Nature remains neutral with regard to jurisdictional claims in published maps and institutional affiliations.

Ready to submit your research? Choose BMC and benefit from:

- fast, convenient online submission

- thorough peer review by experienced researchers in your field

- rapid publication on acceptance

- support for research data, including large and complex data types

- gold Open Access which fosters wider collaboration and increased citations

- maximum visibility for your research: over $100 \mathrm{M}$ website views per year

At BMC, research is always in progress.

Learn more biomedcentral.com/submissions 